\title{
Meson screening masses from lattice QCD with two light and the strange quark
}

\author{
M. Cheng ${ }^{1}$, S. Datta ${ }^{2}$, A. Francis ${ }^{3}$, J. van der Heide ${ }^{3}$, C. Jung ${ }^{4}$, O. Kaczmarek ${ }^{3}$, F. Karsch ${ }^{3,4}$, E. Laermann ${ }^{3}$, R. D. \\ Mawhinney $^{5}$, C. Miao ${ }^{4}$, S. Mukherjee ${ }^{4}$, P. Petreczky ${ }^{4}$, J. Rantaharju ${ }^{3,6}$, C. Schmidt ${ }^{7,8}$ and W. Söldner ${ }^{7,8}$ \\ 1 Physics Division, Lawrence Livermore National Laboratory, Livermore, CA 94550, USA \\ Department of Theoretical Physics, Tata Institute of Fundamental Research, Mumbai 400005, India \\ Fakultät für Physik, Universität Bielefeld, D-33615 Bielefeld, Germany \\ 4 Physics Department, Brookhaven National Laboratory, Upton, NY 11973, USA \\ 5 Physics Department, Columbia University, New York, NY 10027, USA \\ ${ }^{6}$ Department of Physics, University of Helsinki, FI-00014, Finland \\ 7 Frankfurt Institute for Advanced Studies, J.W.Goethe Universität Frankfurt, D-60438 Frankfurt am Main, Germany \\ GSI Helmholtzzentrum für Schwerionenforschung, D-64291 Darmstadt, Germany
}

October 22, 2018

\begin{abstract}
We present results for screening masses of mesons built from light and strange quarks in the temperature range of approximately between $140 \mathrm{MeV}$ to $800 \mathrm{MeV}$. The lattice computations were performed with $2+1$ dynamical light and strange flavors of improved (p4) staggered fermions along a line of constant physics defined by a pion mass of about $220 \mathrm{MeV}$ and a kaon mass of $500 \mathrm{MeV}$. The lattices had temporal extents $N_{\tau}=4,6$ and 8 and aspect ratios of $N_{s} / N_{\tau} \geq 4$. At least up to a temperature of $140 \mathrm{MeV}$ the pseudo-scalar screening mass remains almost equal to the corresponding zero temperature pseudo-scalar (pole) mass. At temperatures around $3 T_{c}\left(T_{c}\right.$ being the transition temperature) the continuum extrapolated pseudo-scalar screening mass approaches very close to the free continuum result of $2 \pi T$ from below. On the other hand, at high temperatures the vector screening mass turns out to be larger than the free continuum value of $2 \pi T$. The pseudo-scalar and the vector screening masses do not become degenerate even for a temperature as high as $4 T_{c}$. Using these mesonic spatial correlation functions we have also investigated the restoration of chiral symmetry and the effective restoration of the axial symmetry. We have found that the vector and the axial-vector screening correlators become degenerate, indicating chiral symmetry restoration, at a temperature which is consistent with the QCD transition temperature obtained in previous studies. On the other hand, the pseudo-scalar and the scalar screening correlators become degenerate only at temperatures larger than $1.3 T_{c}$, indicating that the effective restoration of the axial symmetry takes place at a temperature larger than the QCD transition temperature.
\end{abstract}

PACS. 11.15.Ha , 11.10.Wx, 12.38.Gc, 12.38.Mh

\section{Introduction}

Hadron correlations at finite temperature have been advocated since long as a means to learn about non-perturbative properties of the quark gluon plasma from lattice simulations [1. Studies of the in-medium properties of hadronic excitations provide information about some important lengthscales at high temperatures and give an idea about the relevant degrees of freedom in the plasma and their possible physical effects. Furthermore, these studies also illuminate aspects of the chiral and the effective anomalous $U_{A}(1)$ symmetry restorations in QCD.

Most lattice analyses of hadronic excitations have concentrated on spatial correlation functions. Different from correlators in the temporal direction the physical extent of which is limited by the inverse temperature, a spatial correlation can be studied, in principle, at arbitrarily large distances facilitating the isolation of the lowest excitation. The exponential decay of these spatial correlators defines the so-called screening masses [1].

Physically, the inverse of a screening mass can immediately be interpreted as the spatial distance beyond which the effects of putting a test hadron in the medium are effectively screened. The screening masses $(M)$ need not be identical to the masses $(m)$ defined via the exponential decay of temporal correlators, but they are related to the same spectral function. Thus, amongst other purposes, 
they provide a test bed for model building of the spectral function.

Mesonic spatial correlation functions can be very useful tools to investigate the chiral and effective $U_{A}(1)$ symmetry restorations in QCD. Symmetry restorations demand that certain spatial (and also the temporal) correlation functions become identical at all distances. Of course, this also means that the screening masses of these mesons become degenerate as a symmetry gets restored. However, when one is working with a limited spatial extent, i.e. with relatively smaller volumes, extraction of screening masses from the large distance behavior of the correlators becomes difficult. Even in such cases one can obtain indications of symmetry restorations by studying the degeneracies between certain mesonic spatial correlation functions themselves at relatively shorter distances. Moreover, such symmetry restorations can be studied even without the use of the computationally demanding quark-line disconnected diagrams. As for example, restoration of chiral symmetry can be studied through the degeneracy of the spatial correlation functions belonging to the vector $(\rho)$ and the axial-vector $\left(a_{1}\right)$ channels. It is known [2] that in the limit of mass-less light quarks the 2-point pseudoscalar and scalar correlation functions of the $2+1$ flavor theory are also sensitive to topologically nontrivial configurations similar to the case of a 2 flavor theory. Hence the effective restoration of $U_{A}(1)$ symmetry at high temperature can also be studied using the degeneracy between spatial correlation functions in the pseudo-scalar $(\pi)$ and the iso-triplet scalar $\left(a_{0}\right)$ channels.

Accurate non-perturbative determination of mesonic screening masses also plays an important role in testing the applicability of the dimensionally reduced perturbation theory [3. Within the framework of dimensional reduction at a particular temperature $(T)$ one integrates out all the non-zero bosonic modes and all the fermionic modes of the $3+1$ dimensional theory in order to obtain an effective 3 dimensional theory, for distance scales $R T \gg 1$, consisting of static gauge fields coupled to adjoint scalars. Such a procedure is justified only when the spatial correlation lengths associated with all the fermionic modes in the original $3+1$ dimensional theory are smaller than the largest spatial correlation length associated with the bosonic modes. Thus the applicability of dimensional reduction, at a certain temperature and distance scale, crucially depends on the assumption that the screening masses of all the objects made out of fermionic modes of the original $3+1$ theory are larger than the lowest screening mass associated with objects consisting only of the original gauge fields. In fact, this issue was addressed in Ref. [4] in 4 flavor QCD using the standard staggered action on lattice with temporal extent $N_{\tau}=4$. This study suggests that the pseudo-scalar quark-anti-quark ('pion') correlator gives the largest spatial correlation length (i.e. smallest screening mass) for temperatures as high as $T=3 T_{c}$, $T_{c}$ being the chiral transition temperature, indicating the breakdown of dimensional reduction below that temperature. On the other hand, another recent study [5] in $2+1$ flavor QCD using an improved staggered fermion ac- tion found that the non-perturbative values of the spatial string tension are in good agreement with the prediction of dimensionally reduced perturbation theory 6 right down to $T=1.5 T_{c}$. Thus in order to clarify this issue related to the applicability of dimensional reduction it is necessary to have a detailed study of the meson screening masses in $2+1$ flavor QCD using an improved staggered fermion action and small lattice spacings.

In addition to the test of the applicability of the dimensional reduction framework, improved determinations of mesonic screening masses are also important for checking the accuracy of the prediction of re-summed perturbation theories. In mass-less free field theory the meson screening masses acquire a value of $2 \pi T$ 7 independent of the $J^{P C}$ structure of the interpolating meson operators. The leading order perturbative corrections of $\mathcal{O}\left(g^{2} T\right)$ to this free field value have been calculated using both the dimensional reduction framework 8 and the hard thermal loop framework 9 . It was found that the leading order perturbative correction is positive and equal for all the meson channels. Thus for large temperatures the perturbative results approach the free field value from above in the same way independent of the meson channel.

On the lattice, screening masses have been studied in the quenched approximation and with dynamical quarks, both in Wilson as well as staggered type fermion discretizations (for a review and references see e.g. 10 and references therein). Regarding the general pattern, little differences between quenched and full QCD studies have been observed. Below the transition region differences between masses and screening masses were found to be small, independent of the lattice fermion action. Above the transition mesonic screening masses were found to approach the zero quark mass continuum free field limit of $2 \pi T$ from below. In the temperature range of 2 to 4 times the transition temperature vector screening masses have been closer to this value while pseudo-scalar ones were found to be typically $10 \%$ smaller than the vectors for Wilson quarks and somewhat lower values were reported for the standard staggered action. This is in contrast to the perturbative predictions as mentioned above.

However, most of the studies were performed at fixed lattice spacing and fixed volume $(\mathcal{V})$. More recently, the lattice spacing dependence of screening masses based on the standard staggered action was analyzed in 11. The results show that discretization effects are significant for this choice of action and the pseudo-scalar screening mass at a fixed value of $T \mathcal{V}^{1 / 3}$ approaches the infinite volume free lattice value from below. Preliminary results of a systematic study of volume as well as lattice spacing dependence of the screening masses for quenched improved Wilson fermions on large lattices have shown [12,13 that in this discretization scheme the volume effects increase the screening masses and discretization effects work in the opposite direction. On the other hand, Ref. 14 concludes that finite volume as well as discretization effects are small for quenched overlap fermions, with the vector being close to $2 \pi T$ and the scalar being $10 \%$ lower at $T=2 T_{c}$. 


\begin{tabular}{cc|cc|cc|cc}
\hline & $\phi(\boldsymbol{x})$ & \multicolumn{2}{|c|}{$\Gamma$} & \multicolumn{2}{c|}{$J^{P C}$} & \multicolumn{2}{c}{ states } \\
& & $\mathrm{NO}$ & $\mathrm{O}$ & $\mathrm{NO}$ & $\mathrm{O}$ & $\mathrm{NO}$ & $\mathrm{O}$ \\
\hline $\mathcal{M} 1$ & $(-1)^{x+y+\tau}$ & $\gamma_{3} \gamma_{5}$ & 1 & $0^{-+}$ & $0^{++}$ & $\pi_{2}$ & $a_{0}$ \\
$\mathcal{M} 2$ & 1 & $\gamma_{5}$ & $\gamma_{3}$ & $0^{-+}$ & $0^{+-}$ & $\pi$ & - \\
$\mathcal{M} 3$ & $(-1)^{y+\tau}$ & $\gamma_{1} \gamma_{3}$ & $\gamma_{1} \gamma_{5}$ & $1^{--}$ & $1^{++}$ & $\rho_{2}^{\mathcal{T}}$ & $a_{1}^{\mathcal{T}}$ \\
$\mathcal{M} 4$ & $(-1)^{x+\tau}$ & $\gamma_{2} \gamma_{3}$ & $\gamma_{2} \gamma_{5}$ & $1^{--}$ & $1^{++}$ & $\rho_{2}^{\mathcal{T}}$ & $a_{1}^{\mathcal{T}}$ \\
$\mathcal{M} 5$ & $(-1)^{x+y}$ & $\gamma_{4} \gamma_{3}$ & $\gamma_{4} \gamma_{5}$ & $1^{--}$ & $1^{++}$ & $\rho_{2}^{\mathcal{L}}$ & $a_{1}^{\mathcal{L}}$ \\
$\mathcal{M} 6$ & $(-1)^{x}$ & $\gamma_{1}$ & $\gamma_{2} \gamma_{4}$ & $1^{--}$ & $1^{+-}$ & $\rho_{1}^{\mathcal{T}}$ & $b_{1}^{\mathcal{T}}$ \\
$\mathcal{M} 7$ & $(-1)^{y}$ & $\gamma_{2}$ & $\gamma_{1} \gamma_{4}$ & $1^{--}$ & $1^{+-}$ & $\rho_{1}^{\mathcal{T}}$ & $b_{1}^{\mathcal{T}}$ \\
$\mathcal{M} 8$ & $(-1)^{\tau}$ & $\gamma_{4}$ & $\gamma_{1} \gamma_{2}$ & $1^{--}$ & $1^{+-}$ & $\rho_{1}^{\mathcal{L}}$ & $b_{1}^{\mathcal{L}}$ \\
\hline
\end{tabular}

Table 1. The list of meson operators studied in this work. States associated with the non-oscillating and the oscillating part of the screening correlators are designated by the identifiers NO and O respectively. Particle assignments of the corresponding states are given only for the $u-d$ flavor combination.

Here we present lattice results for meson screening masses in $2+1$ flavor QCD using improved p4 15] staggered fermions with the zero temperature Goldstone pion mass of $220 \mathrm{MeV}$ and the zero temperature kaon mass of $500 \mathrm{MeV}$. The temperature ranges from about $140 \mathrm{MeV}$ to maximally $820 \mathrm{MeV}$. Our study is performed at three different sets of the lattice spacings corresponding to $N_{\tau}=$ 4, 6 and 8, which allows to address discretization effects.

The paper is organized such that after presenting the numerical set-up in section 2 we briefly discuss discretization and finite volume effects as they arise, for our choice of action, in semi-analytical computations in the free case. This provides the background on which we present and discuss our results on screening masses in section 4 They have been determined from spatial correlators of operators built with degenerate up $(u)$ and down $(d)$ quarks as well as with strange $(s)$ quarks with the same masses as in the generation of the configurations. Preliminary results have been presented earlier in [13,16.

\section{Numerical set-up}

In the staggered lattice formulation of QCD meson operators can be defined [17] as $\mathcal{M}=\bar{\psi}\left(\Gamma^{D} \otimes \Gamma^{F}\right) \psi$, where the fermion field $\psi$ has four Dirac- and four flavor/tastecomponents and is defined on a coarse lattice with lattice spacing $2 a$. The matrices $\Gamma^{D}$ and $\Gamma^{F}$ are products of $\gamma$-matrices and generate the spin-flavor/taste structure of the corresponding meson. Here we are interested in local meson operators, for which $\Gamma^{D}=\Gamma^{F} \equiv \Gamma$. In terms of staggered fermion fields $(\boldsymbol{x})$ which are defined at the points $\boldsymbol{x}=(x, y, z, \tau)$ of a fine lattice with spacing $a$, these local meson operators can be written as $\mathcal{M}(\boldsymbol{x})=\tilde{\phi}(\boldsymbol{x}) \bar{\chi}(\boldsymbol{x})(\boldsymbol{x})$, where $\tilde{\phi}(\boldsymbol{x})$ is a phase factor depending on the choice of $\Gamma$.

In this work we study mesons built from $\bar{u} d, \bar{u} s$ and $\bar{s} s$ flavor combinations. For the flavor non-singlet combinations $\bar{u} d$ and $\bar{u} s$ the correlation function consists solely of the quark-line connected part. However, for the flavor singlet $\bar{s} s$ flavor combination we only use the connected part of the correlation function and neglect the computationally demanding quark-line disconnected part. The connected part of the staggered meson screening correlator, projected to zero transverse momentum, $\boldsymbol{p}_{\perp}=\left(p_{x}, p_{y}\right)$, and to zero (boson) Matsubara frequency, $\omega_{n}$, is obtained as

$$
C(z)=\sum_{x, y, \tau} \phi(\boldsymbol{x})\left\langle\left(M_{\mathbf{0 x}}^{-1}\right)^{\dagger} M_{\mathbf{0 x}}^{-1}\right\rangle,
$$

where $M_{\mathbf{0 x}}^{-1}$ is the full staggered propagator from $\mathbf{0}$ to $\boldsymbol{x}$. Since a staggered fermion meson correlator, in general, contains two different mesons with opposite parity [18 the correlator is parametrized as

$$
\begin{aligned}
C(z)= & A_{N O} \cosh \left[M_{-}\left(z-\frac{N_{s}}{2}\right)\right] \\
& -(-1)^{z} A_{O} \cosh \left[M_{+}\left(z-\frac{N_{s}}{2}\right)\right] .
\end{aligned}
$$

Different from e.g. [18, 19] we have chosen the phase factor in Eq. (2) as $\phi(\boldsymbol{x})=-(-1)^{x+y+\tau} \tilde{\phi}(\boldsymbol{x})$. According to this convention $M_{-}\left(M_{+}\right)$corresponds to the screening mass of the lightest negative (positive) parity state 1 and comes as the non-oscillating, NO (oscillating, O), part of the screening correlator. For instance, in this convention the Goldstone pion comes as the non-oscillating part of the screening correlator. Note that both amplitudes are positive [19], $A_{N O}, A_{O} \geq 0$.

For staggered fermions there are 8 possible local meson operators 18 which are listed in Table 1, together with the corresponding phase factors $\phi(\boldsymbol{x})$. We separate between the transverse $(\mathcal{T})$ and longitudinal $(\mathcal{L})$ polarizations of vector and axial-vector correlators. At $T=0$ the rotational symmetry group of the lattice corresponds to the continuum $O(3)$. For spatial correlators, however, at non-zero temperature this symmetry breaks down to $O(2) \times Z(2)$ 20. Thus, the longitudinal states are allowed to acquire screening masses different from the transverse ones.

For the present study we have analyzed the gauge configurations generated by means of the RHMC algorithm [21] by the RBC-Bielefeld [5, 22] and the HotQCD [23] collaboration using the $\mathrm{p} 4$ staggered action. These configurations were generated for 2 degenerate dynamical light quarks and 1 heavier dynamical strange quark along the Line of Constant Physics (LCP). The LCP is obtained by tuning the bare quark masses such that at zero temperature the (Goldstone) pion mass $m_{\pi}$ is approximately 220 $\mathrm{MeV}$ and the kaon mass $m_{K}$ equals $500 \mathrm{MeV}$. As in [22, relative scales have been obtained from the Sommer $r_{0}$ parameter 24], absolute ones from bottomonium splittings 25] resulting in $r_{0}=0.469 \mathrm{fm}$ in the continuum limit for physical quark masses. For more details about the simulations and scale settings confer Ref. 22. The values of coupling constant and bare light quark mass in lattice units at which these configurations were generated are summarized

1 For the screening states we keep, as usual, the $J^{P C}$ assignments as they would emerge, for the same operators and correlation functions, in the zero temperature limit. 


\begin{tabular}{|c|c|c|c|c|c|c|c|}
\hline \multirow[b]{2}{*}{$\beta$} & \multirow[b]{2}{*}{$m_{l}$} & \multicolumn{2}{|c|}{$16^{3} \times 4$} & \multicolumn{2}{|c|}{$24^{3} \times 6$} & \multicolumn{2}{|c|}{$32^{3} \times 8$} \\
\hline & & $T$ & conf. & $T$ & conf. & $T$ & conf. \\
\hline 3.290 & 0.00650 & 192 & 1600 & & & & \\
\hline 3.320 & 0.00650 & 201 & 1600 & & & & \\
\hline 3.351 & 0.00591 & 218 & 800 & 145 & 984 & & \\
\hline 3.382 & 0.00520 & 234 & 600 & & & & \\
\hline 3.410 & 0.00412 & 263 & 1000 & 175 & 584 & & \\
\hline 3.430 & 0.00370 & & & 186 & 514 & 139 & 783 \\
\hline 3.445 & 0.00344 & & & 197 & 949 & & \\
\hline 3.455 & 0.00329 & & & 200 & 438 & & \\
\hline 3.460 & 0.00313 & 304 & 600 & 203 & 406 & & \\
\hline 3.490 & 0.00290 & & & 226 & 824 & & \\
\hline 3.500 & 0.00253 & & & & & 175 & 590 \\
\hline 3.510 & 0.00259 & & & 240 & 249 & & \\
\hline 3.540 & 0.00240 & 388 & 400 & 259 & 432 & & \\
\hline 3.570 & 0.00212 & 422 & 1000 & 281 & 385 & 211 & 610 \\
\hline 3.585 & 0.00192 & & & & & 219 & 460 \\
\hline 3.630 & 0.00170 & 489 & 800 & 326 & 399 & & \\
\hline 3.690 & 0.00150 & 547 & 500 & 365 & 450 & & \\
\hline 3.760 & 0.00130 & 637 & 1000 & 424 & 320 & 318 & 477 \\
\hline $3.820^{*}$ & 0.00110 & 710 & 700 & & & & \\
\hline $3.920^{*}$ & 0.00092 & 799 & 3280 & 532 & 497 & & \\
\hline & & 32 & $\times 4$ & 32 & $\times 6$ & & \\
\hline 3.820 & 0.00125 & & & 480 & 742 & 361 & 509 \\
\hline 3.920 & 0.00110 & 820 & 550 & 547 & 834 & 410 & 501 \\
\hline 4.000 & 0.00092 & & & 633 & 890 & 475 & 899 \\
\hline 4.080 & 0.00081 & & & 727 & 580 & 549 & 593 \\
\hline 4.200 & 0.00068 & & & & & 652 & 300 \\
\hline
\end{tabular}

Table 2. Coupling constants $\beta$ and light quark masses $m_{l}$, temperatures $T$ in $\mathrm{MeV}$ and the number of configurations of the given sizes on which screening masses were computed. The strange quarks have always been ten times heavier than the light ones, $m_{s}=10 m_{l}$. At the $\beta$ values marked with a star we analyzed configurations which were generated in an early stage of the project at quark masses slightly off the LCP.

in Table 2. The bare strange quark mass has always been 10 times larger, $m_{s}=10 m_{l}$. The table also contains the temperature values and lists the number of configurations which were used for the present analysis. The configurations were separated by 10 trajectories. Autocorrelation times were estimated from the point-to-point spatial correlation functions at intermediate to large separations $z$ from source and sink. The largest autocorrelations were observed in the lightest pseudo-scalar channel, $\mathcal{M} 2$, and turned out to be of $\mathcal{O}(10)$ configurations, i.e. $\mathcal{O}(100)$ trajectories, in the vicinity of the transition and dropping quickly away from it.

\section{Free case}

Lattice results are in general affected by discretization as well as by finite volume effects. At fixed temperature these are parametrized through the temporal extent of the lattice, $a T=1 / N_{\tau}$, and the aspect ratio, $T \mathcal{V}^{1 / 3}=N_{s} / N_{\tau}$, respectively. Discretization and finite volume effects are readily computed semi-analytically in the non-interacting theory. While in this case both effects are expected to be larger than in the interacting theory [12,13] these computations give at least hints about the sign and the order of magnitude of the lattice distortions. The free case is also the infinite temperature limit of the interacting theory. In this limit the spectral function underlying both, temporal as well as spatial correlation functions, solely consists of a cut in the complex frequency plane. The free case thus provides a test example for screening masses arising from a cut.

In the free case at vanishing quark mass only states of one parity contribute to the correlation functions. In channels $\mathcal{M} 2$ and $\mathcal{M} 5$ to $\mathcal{M} 7$ these are states with negative parity while in the remaining channels the contributing states have positive parity. In the latter case an additional phase factor $-(-1)^{z}$ has been applied to $C(z)$, see Eq.(2) and Table 1, such that for all channels the effective mass can be defined as

$$
a M_{e f f}(z)=-\ln \left[\frac{C(z+1)}{C(z)}\right] .
$$

With this sign convention the correlation functions of all channels are degenerate at zero quark mass for all values of $N_{\tau}$ and aspect ratios $N_{s} / N_{\tau} \geq 4$. At large $z$ the spatial meson correlators start oscillating. These oscillations originate from a contribution $\sim(-)^{z} \cdot$ constant (in our phase convention) which vanishes in the thermodynamic limit $N_{s} \rightarrow \infty$.

In Fig. 1(a) we show $M_{\text {eff }}(z)$ as a function of the inverse separation in units of temperature, $1 /(z T)$, for $\mathrm{p} 4$ fermions at various values of the temporal lattice extent. As can be seen, for the improved p4 action there is practically no $N_{\tau}$ dependence for $z T \geq 1$. On the other hand, for the unimproved, standard staggered action the discretization errors are significantly larger, as is shown in Fig. 1(b) for comparison.

However, for both the p4 and the standard staggered action the effective mass from a point correlator is strongly dependent on $z$, and the value $2 \pi T$ is obtained only in the limit of infinite $z$. This is in accord with the continuum, non-lattice calculation of the spatial pion correlation function [7] where this value is also only reached in the limit $z \rightarrow \infty$ of the continuum equivalent of Eq.(3). The $z$ dependence of the effective screening mass is due to the cut in the spectral function. Because of contributions from quarks at all momenta, $p_{x}, p_{y}$ and all Matsubara frequencies, $\omega_{n}=\pi T(2 n+1)$ which die out only in the limit $z \rightarrow \infty$ the effective screening mass does not develop a plateau. This is strictly true for effective masses calculated from point-to-point correlation functions. Extended sources, for instance wall sources, suppress contributions from higher quark momenta and thus may lead to a plateau like behavior in effective mass plots, at least in a limited $z$ interval. Since infinite separations can only be achieved in the thermodynamic limit, Fig. 1 suggests that the screening masses determined from lattice simulations may have significant dependence on the maximum available distance along which the correlation functions were measured. This can be considered as a finite volume effect, although an indirect one as for aspect ratios larger 


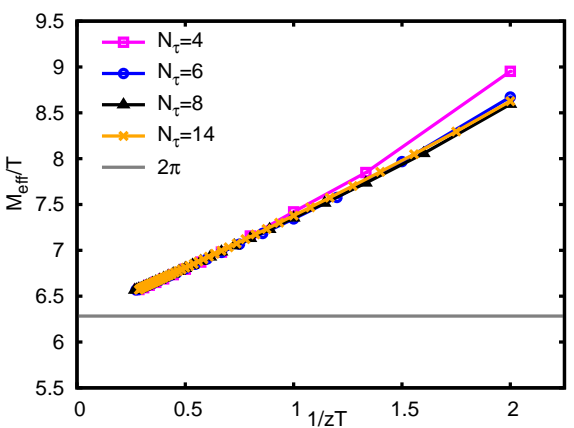

(a)

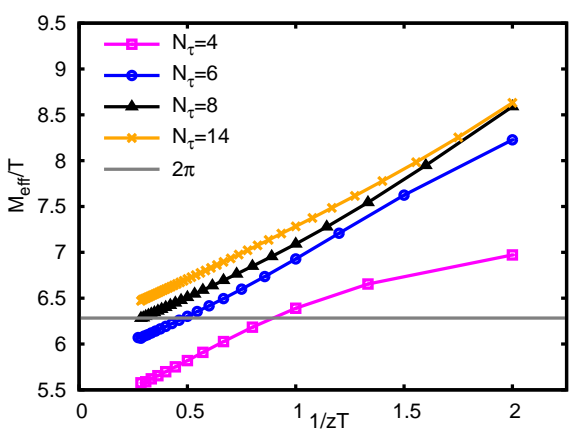

(b)

Fig. 1. Dependence of the effective screening mass, Eq.(3), on $N_{\tau}$ and on the separation $z T$, obtained from point correlators for a free theory with the p4 staggered action (a) and with standard staggered fermions (b).

than 3 there is practically no volume dependence of the free results other than through the limited $z$ range that is available. As a corollary, we note that the absence of a plateau in effective masses extracted from point correlators is a necessary condition to the verification of free field behavior in screening masses.

\section{Results}

The following results on screening masses of mesonic states built from light quarks have been obtained on dynamical $N_{F}=2+1$ gauge field configurations. The analysis was performed on lattices of size $16^{3} \times 4,24^{3} \times 6$ and $32^{3} \times 8,5$, 22,23 accompanied by some cross-checks on available high temperature lattices with larger aspect ratios at $N_{\tau}=4$ and 6, (see Table 2). On all those configurations we computed spatial correlations of meson operators (see Table 1) made out of degenerate $u$ and $d$ quarks. In addition we studied mesons with $\bar{u} s$ and $\bar{s} s$ flavor content on the lattices with temporal extent $N_{\tau}=4$ and 6 . In all cases the quark masses of the quark propagators have been chosen to be the same as in the quark determinant, i.e. in the generation of the configurations. On all lattices we computed point-to-point correlation functions. Subsequently, for most of the $N_{\tau}=4$ and 6 configurations, and some of the ones at $N_{\tau}=8$, (see the tables in the appendix) we also analyzed correlators from wall sources.

In general, in a staggered fermion meson (screening) correlator a meson is accompanied by an opposite parity meson, see Eq. (2). However, in the present study, in most channels we have seen indications for the presence of both of these mesons at most at the lowest temperatures. With increasing temperature, the contribution of one of the two mesons died out very fast. Hence, in most cases we found the signature of only one of the states. Specifically:

- The $\mathcal{M} 1$ channel is dominated by the oscillating positive parity scalar. A non-oscillating negative parity contribution due to a pseudo-scalar state could not be isolated. We will denote the screening masses in this scalar channel as $M_{S C}$, keeping in mind that at least at zero temperature the scalar channel contains two pseudo-scalar meson contributions which may become particularly important in the presence of taste violations [26].

- In the $\mathcal{M} 2$ channel we have not seen any oscillating contribution. This channel is dominated by the Goldstone pseudo-scalar at all temperatures. The screening masses in this channel will be denoted by $M_{P S}$.

- The $\mathcal{M} 3$ and $\mathcal{M} 4$ point correlators are noisy at low temperature but the signal-to-noise ratio improves greatly with rising $T$. Wall source correlators delivered suitable signals at all temperatures. In all fits we have found stable signatures only of positive parity axial-vectors. The screening masses of the axial-vectors, denoted by $M_{A V}$ later, coming from these two channels are degenerate

- In $\mathcal{M} 6$ and $\mathcal{M} 7$ we found only the negative parity vector states. The signal-to-noise ratios were similar to the ones seen in $\mathcal{M} 3$ and $\mathcal{M} 4$. The screening masses of these states, $M_{V}$, coming from these two channels are found to be degenerate for all temperatures.

- Channel $\mathcal{M} 5$ as well as $\mathcal{M} 8$ were found to be very noisy in point correlators and reasonable signals could only be obtained from them at the highest temperatures. The wall correlators are at low temperature dominated by the axialvector in $\mathcal{M} 5$ channel and the vector in $\mathcal{M} 8$ channel. At high temperature, independent of the source we found signatures of vector as well as axial-vector states in both channels.

- These observations hold for all flavor combinations where, however, the signal-to-noise ratio is in general slightly better for the heavier combinations.

- Contrary to the free case, in none of the correlation functions a contribution constant in $z$ was observed.

In all cases where acceptable signal-to-noise ratios were obtained within our statistics, the effective masses $M_{\text {eff }}(z)$ from point-to-point correlators show reasonable plateaux in $z$ within errors. This is particularly true at high temperature where we also analyzed lattices with aspect ratios larger than 4 to access larger $z T$ values. There is a slight trend in the effective masses to decrease with increasing $z$, but in none of the channels the free field behavior as depicted in Fig. 1(a) could be verified.

Subsequently we analyzed spatial meson correlators from wall sources built along the $x, y, \tau$ directions. As 


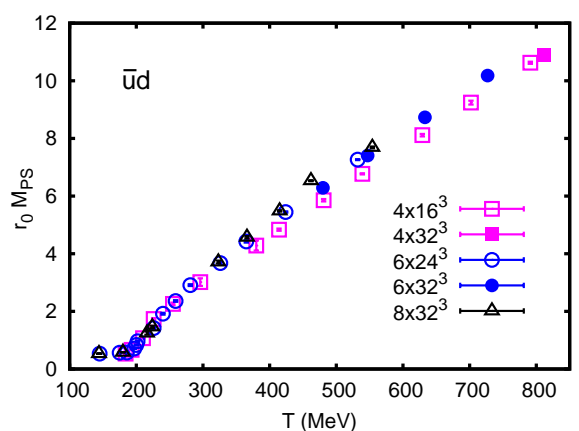

(a)

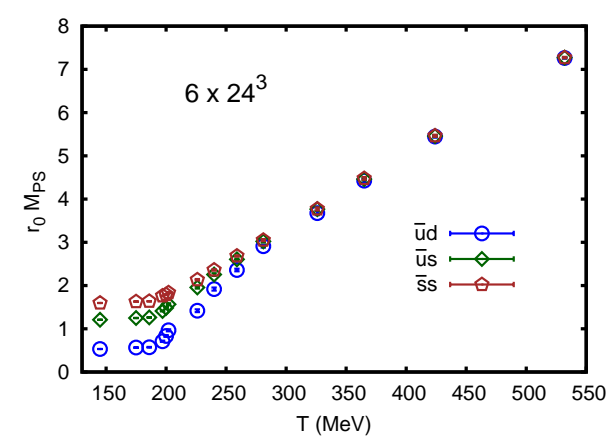

(b)

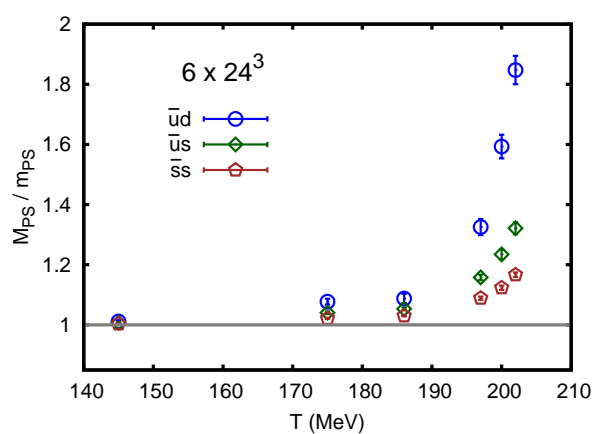

(c)

Fig. 2. (a) The pseudo-scalar screening masses in units of $r_{0}$ for $N_{\tau}=4,6$ and 8 . The $N_{\tau}=4$ temperatures have been shifted by $-8 \mathrm{MeV}$ and the $N_{\tau}=8$ ones by $+5 \mathrm{MeV}$, see text. (b) Flavor dependence of the pseudo-scalar screening masses at $N_{\tau}=6$. (c) Ratio of the pseudo-scalar screening masses at $N_{\tau}=6$ to the corresponding zero temperature masses determined at the same couplings along the line of constant physics with $m_{\pi} \simeq 220 \mathrm{MeV}$ and $m_{K} \simeq 500 \mathrm{MeV}$.

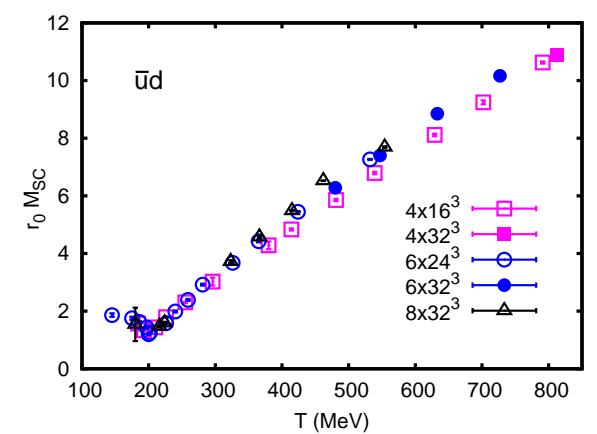

(a)

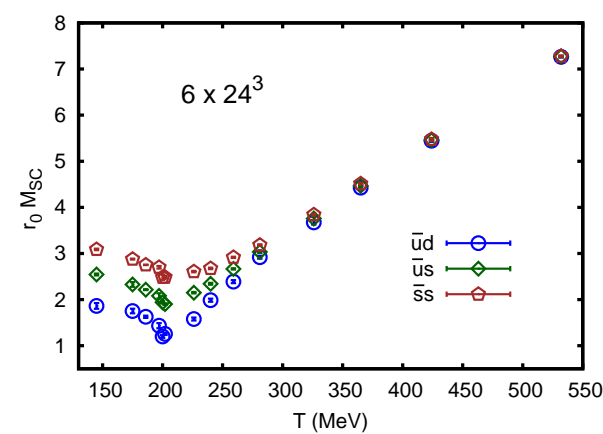

(b)

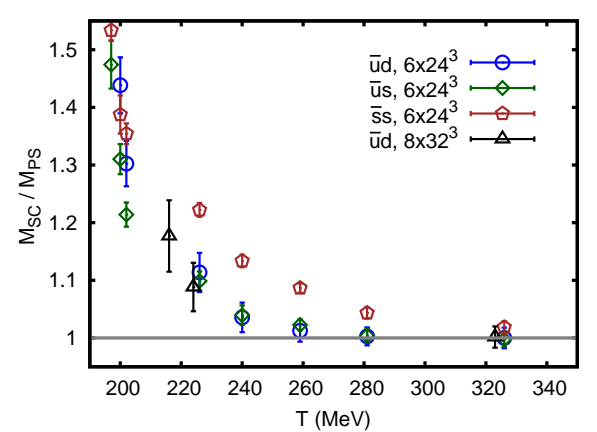

(c)

Fig. 3. (a) The scalar screening masses in units of $r_{0}$ for $N_{\tau}=4,6$ and 8 . Temperatures have been shifted as in Fig. 2(a) (b) Flavor dependence of the scalar screening masses at $N_{\tau}=6$. (c) Ratio of the scalar to the pseudo-scalar screening mass at high temperature. The data is from the $N_{\tau}=6$ and 8 lattices for the $\bar{u} d$ flavor combination and from $N_{\tau}=6$ for $\bar{u} s$ and $\bar{s} s$.

pointed out in the previous section, effective masses from wall source correlators potentially pretend the presence of genuine bound states even in the free case because contributions from free quarks with higher momenta are suppressed by construction. However, the point-to-point correlation functions have already indicated the absence of a substantial contribution from a free quark cut at the temperatures investigated.

The effective masses from the wall source correlators in general approach plateaux from below, as at zero temperature. For the scalar and the pseudo-scalar channels the error bars on the screening masses are of the same magnitude as for the point correlators. The wall source masses tend to be slightly lower than the point source ones but the differences are at the one $\sigma$ margin. Since the scalar and pseudo-scalar data set for point sources is most complete we will plot point source results in the following, for wall source numbers we refer to the tables in the appendix. In the vector and axial-vector channels the signal-to-noise ratio is much better for the wall sources, in particular at low temperatures. At high temperatures the differences between wall and point source results are again small, with somewhat smaller error bars on the wall source results. Except for the $N_{\tau}=8$ lattices, we will therefore plot and discuss wall source results for those channels below.

Independent of the source, the screening mass values which will be quoted in the following have been obtained from fits to the correlators with a minimum distance $z_{\text {min }}$ from source and sink of about $z_{\min } T \simeq 1$, except for the high temperature checks on lattices with aspect ratios larger than 4 where larger separations up to $z_{m i n} T \simeq 2$ could be accessed. Errors were determined by jack-knifing the fits.

For the purpose of showing our data as a function of a common temperature scale, throughout this paper the data for $N_{\tau}=4$ has been shifted in temperature by a value of $\Delta T=-8 \mathrm{MeV}$ and the one for $N_{\tau}=8$ by $\Delta T=+5$ $\mathrm{MeV}$ to properly account for the $N_{\tau}$ dependence of the transition region [23. Also throughout this paper we will use the chiral crossover temperature of $T_{c} \simeq 196 \mathrm{MeV}$ as our reference transition temperature. This corresponds to the chiral crossover temperature, determined from the peak of the disconnected chiral susceptibility, for $6 \times 24^{3}$ lattices with $m_{\pi} \simeq 220 \mathrm{MeV}$ and $m_{K} \simeq 500 \mathrm{MeV}$ [23]. 
In Fig. 2(a) we show our results for $M_{P S}$, in the $\bar{u} d$ flavor combination, in units of $r_{0}$ for all $N_{\tau}$ values of 4,6 and 8 as a function of temperature. The pseudo-scalar screening masses stay constant to a high degree of accuracy below the transition region and rise rapidly above. For instance, at a temperature of about $250 \mathrm{MeV}$ the (lightest) pseudo-scalar mass has already reached a value of $\simeq 3.5 T$ and at $T \simeq 400 \mathrm{MeV}$ the value has increased to $\simeq 5.25 T$ in temperature units. The rapid rise of $M_{P S} / T$ indicates that in this temperature range the scale for the pseudoscalar screening mass is not set solely by the temperature.

In Fig. 2(b) we compare the different flavor channels for $N_{\tau}=6$. Below the transition region, for all flavor combinations the pseudo-scalar screening masses stay approximately constant in temperature and are markedly different from each other, in accord with the mass differences at zero temperature. Above the transition region the differences between the flavor channels become smaller with rising temperature and practically vanish at around $300 \mathrm{MeV}$.

The differences between zero temperature masses and finite temperature screening masses in the confined phase are inspected more closely in Fig. 2(c) As can be seen, the ratio of $M_{P S}$ to $m_{P S}$, the ordinary zero temperature Goldstone pion masses determined at the same couplings along the line of constant physics, starts differing from one for temperatures $T \gtrsim 170 \mathrm{MeV}$. This holds true for all the three flavor combinations $\bar{u} d, \bar{u} s$ and $\bar{s} s$. However, in the range $170 \mathrm{MeV} \leq T \lesssim 190 \mathrm{MeV}$, the differences are distinct but only of the order of $5-10 \%$ slightly depending on the quark mass.

In Fig. 3(a) we show the temperature dependence of $M_{S C}$, normalized by $r_{0}$, in the $\bar{u} d$ flavor combination for our lattices with temporal extents 4,6 and 8. Note that $M_{S C}$ decreases with $T$ at low temperature before it starts to rise rapidly at $200 \mathrm{MeV}$. This is seen more clearly in Fig. 3(b) where we plot $M_{S C}$ from the $N_{\tau}=6$ lattices for all flavor combinations. Above $200 \mathrm{MeV}$ the screening mass differences between the flavor channels persist up to about $325 \mathrm{MeV}$ and vanish at higher temperature.

In the presence of sufficiently strong taste violations and at small enough Goldstone boson masses, at least at zero temperature the scalar flavor non-singlet channel, $a_{0}$, may receive its dominant contribution from an unphysical two-Goldstone boson threshold [26. However, in our case of screening correlators we observe that at temperatures in the confined phase $M_{S C}$ as obtained from fits to the data is significantly larger than two times the Goldstone boson screening mass. At the same time $M_{S C}$ is lower than twice the Kaon mass as well as the $\pi \eta$ threshold where the $\eta$ mass was estimated with the Gell-MannOkubo formula $m_{\eta}^{2}=\left(4 m_{K}^{2}-m_{\pi}^{2}\right) / 3$. This indicates that here indeed $M_{S C}$ denotes the screening mass of a genuine scalar. We then compare this scalar screening mass with the pseudo-scalar one at temperatures above $200 \mathrm{MeV}$ in Fig. 3(c), The figure highlights that the differences between the two gradually disappear with rising temperature, leading to degeneracy, but only above a temperature of $250 \mathrm{MeV}$. Note that the differences for the channels containing light quarks, i.e. the one with the light $\bar{u} d$ content as well as the heavier $\bar{u} s$ combination, agree with each other to a good degree of accuracy suggesting that those differences survive in the light quark chiral limit. The non-degeneracy of scalar and pseudo-scalar screening mass still observed at temperatures up to at least 250 $\mathrm{MeV}$ thus indicates that the anomalous $U_{A}(1)$ symmetry is not effectively restored at this temperature. The nonzero mass splitting $\left(M_{S C}-M_{P S}\right)$ for the $\bar{s} s$ sector for $250 \mathrm{MeV} \lesssim T \lesssim 325 \mathrm{MeV}$ is most likely due to larger explicit $U_{A}(1)$ breaking in presence of the larger strange quark masses. This is also supported by the fact that also in this sector this mass splitting vanishes for $T \gtrsim 325$ $\mathrm{MeV}$ which, as mentioned before, is the temperature range where the difference between the light and strange quark masses become unimportant.

In Fig. 4(a) the results for $M_{V}$ at different $N_{\tau}$ values are shown. At temperatures below the transition the vector screening mass $M_{V}$ in physical units is roughly constant in $T$, while for temperatures larger than about 250 $\mathrm{MeV}$ its ratio with $T, M_{V} / T$, is approximately constant over the investigated temperature range. This indicates that different from the pseudo-scalar and scalar channels the temperature sets the scale for the vector screening masses already slightly above the transition. The ratio $M_{V} / T$ is slightly larger than the continuum free result at infinite separation. However, a comparison with the free lattice result in the region around $z T \simeq 1$ which typically is the lower boundary of the fit window shows that the data are about $20 \%$ lower than this number. Moreover, even at the highest temperatures available the effective masses from point correlators do not show the $z$ dependence as in the free case.

Fig. 4(b) shows the flavor dependence of the vector screening masses as a function of temperature for $N_{\tau}=6$. At low temperatures the screening masses are ordered according to the strange quark content whereas already at a temperature of $T \simeq 225 \mathrm{MeV}$, the vector screening masses are flavor independent, supporting that for the vector channel it is the temperature which sets the scale.

We also inspect the degeneracy of $M_{P S}$ and $M_{V}$, which is expected at least in the limit of infinite temperature. In Fig. 4(c) we show the ratio $M_{P S} / M_{V}$ for the lightest flavor combination, noting that the ratio is independent of the quark flavor for temperatures larger than $300 \mathrm{MeV}$. Also for this ratio, the results from the $N_{\tau}=4$ lattices are somewhat lower than those from the other two lattice sets with $N_{\tau}=6$ and 8 , which are in good agreement with each other. Overall we find that $M_{P S} / M_{V}$ is statistically significantly lower than 1 even at temperatures $T \gtrsim 500$ $\mathrm{MeV}$. Taking the $N_{\tau}=8$ result at $T=550 \mathrm{MeV}$ at face value the difference from unity is about $9 \%$. There is, however, a slight trend that the ratio rises with temperature.

Fig. [5]summarizes our results for the axial-vector screening masses. Below the transition the screening masses are ordered according to their strangeness content but above the flavor dependence is weak, Fig. 5(b). Note that below $200 \mathrm{MeV}$ the axial-vector screening mass is decreasing with rising temperature leading to a decrease in its 


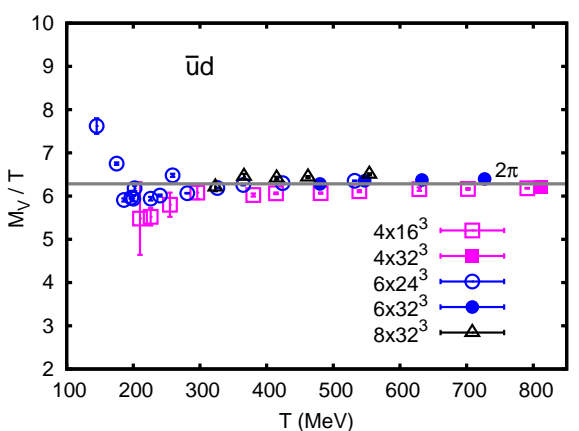

(a)

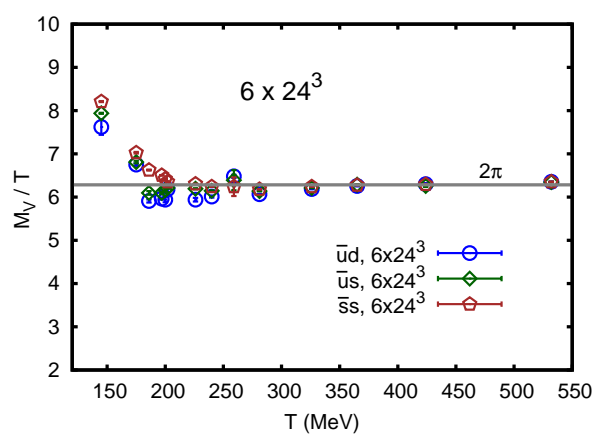

(b)

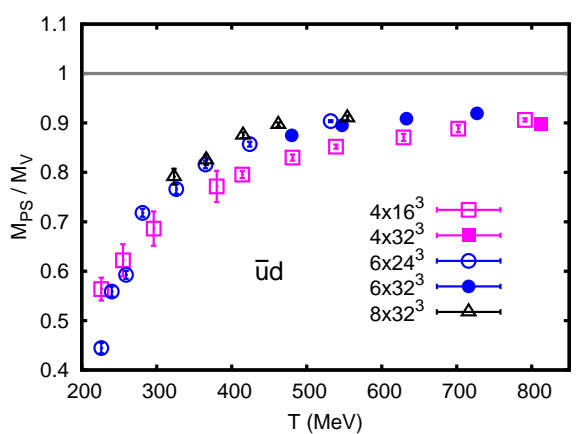

(c)

Fig. 4. (a) Temperature and lattice spacing dependence of the vector screening mass, $M_{V} / T$, in units of temperature for the $\bar{u} d$ flavor combination. Temperatures have been shifted as in Fig. 2(a). (b) Flavor dependence of the vector screening masses at $N_{\tau}=6$. (c) Ratio of the pseudo-scalar to vector screening mass in the $\bar{u} d$ flavor combination.

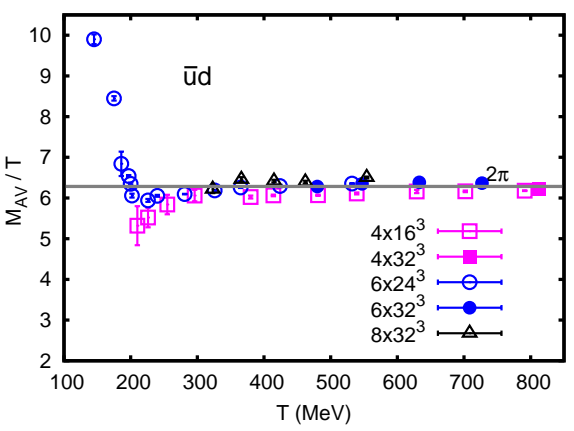

(a)

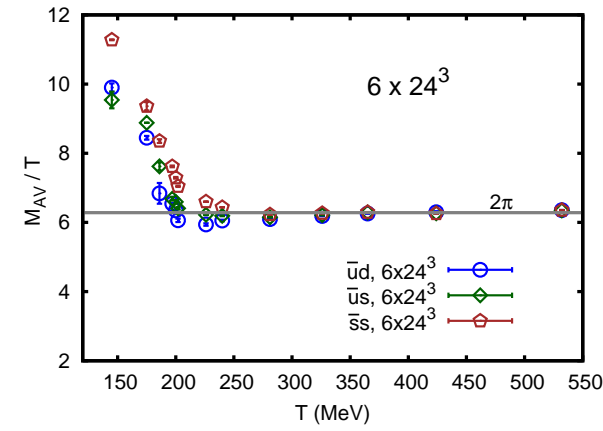

(b)

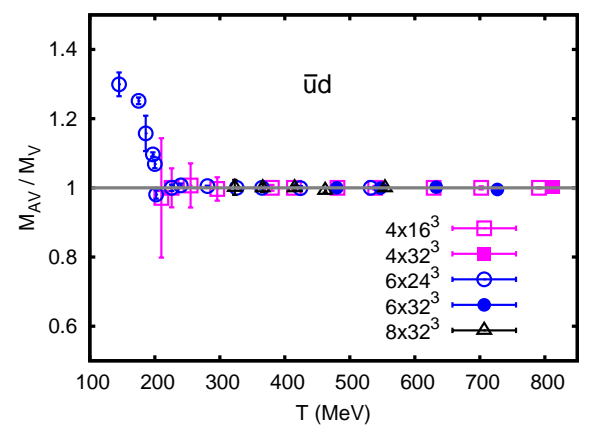

(c)

Fig. 5. The axial-vector screening mass: (a) Temperature and lattice spacing dependence in the $\bar{u} d$ flavor combination, as in Fig. 4(a) (b) Flavor dependence at $N_{\tau}=6$. (c) Ratio to the vector screening mass, in the $\bar{u} d$ flavor combination.

ratio to the vector screening mass, Fig. 5(c). This ratio becomes compatible with 1 around $T \approx 200 \mathrm{MeV}$. Thus within errors axial-vector and vector screening masses become degenerate at this temperature and remain so at all larger temperatures. Although the quark masses are not at the chiral limit the restoration of chiral symmetry is thus also reflected in spectrum results.

The longitudinal $\mathcal{M} 5$ and $\mathcal{M} 8$ channels in general have the largest error bars, also when wall sources are used. In the wall source correlators at temperatures below the transition, $\mathcal{M} 5$ is dominated by an axial-vector while in $\mathcal{M} 8$ only a vector could be isolated. Both states are, within errors, degenerate with the transverse ones. At high temperature, in wall as well as point correlators, where available, the vector contribution to $\mathcal{M} 5$ has the same screening mass as the axial-vector contribution to $\mathcal{M} 8, M_{-}(\mathcal{M} 5)=$ $M_{+}(\mathcal{M} 8)$. Both values are about $5 \%$ larger than the degenerate transverse screening masses, $M_{A V}$ or $M_{V}$. Similarly, the axial-vector contribution to $\mathcal{M} 5$ is equal to the vector contribution to $\mathcal{M} 8, M_{+}(\mathcal{M} 5)=M_{-}(\mathcal{M} 8)$. These screening masses turn out to be roughly $20 \%$ larger than $M_{V}$. As already noted in Section 2 due to the breakdown of $O(3)$ rotational symmetry to $O(2) \times Z(2)$ for spatial correlators at finite temperature [20] the longitudinally polarized (axial-) vector screening states in $\mathcal{M} 5$ and $\mathcal{M} 8$ do not need to be degenerate with the transverse polarizations of the $\mathrm{V}$ and $\mathrm{AV}$ channels. However, an explanation for the ordering observed here for the first time is not known to us.

\section{Summary and discussion}

Utilizing the gauge configurations generated by the RBCBielefeld [5,22] and the HotQCD [23] collaborations using the improved $\mathrm{p} 4$ fermion action along a line of constant physics, determined by $m_{\pi} \simeq 220 \mathrm{MeV}$ and $m_{K} \simeq 500$ $\mathrm{MeV}$, we have investigated the screening masses of mesons in $2+1$ flavor QCD from 8 different local meson operators listed in Table 1 In order to address discretization errors the analysis was carried out at three different values of the temporal lattice extent, $N_{\tau}=4,6$ and 8. Although for most of our analyses we have used lattices with volumes $T \mathcal{V}^{1 / 3}=4$, at high temperatures we have also analyzed some lattices with larger volumes in order to access larger separations. Moreover, the quark mass dependence was studied by analyzing screening correlators for three different quark flavor combinations, viz. $\bar{u} d, \bar{u} s$ and $\bar{s} s$. In order to gain some idea about the systematics we have computed correlation functions using both point and wall sources. 


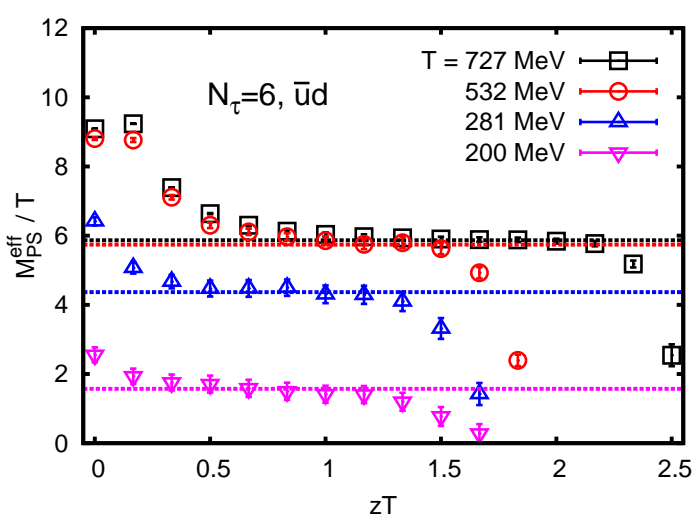

Fig. 6. Effective screening masses in the pseudo-scalar channel as a function of the distance $z T$ for some illustrative values of temperatures. The dashed lines indicate the values of the respective screening masses obtained from cosh fits to the correlators. Except for the highest temperature, where the correlator was measured using wall source, all the correlators were obtained using point sources on $N_{\tau}=6$ lattices for the $\bar{u} d$ flavor combination.

As discussed in Section 3, the presence of a cut in the spectral function will be manifested through a decreasing effective screening mass (coming from point source correlation function) as a function of increasing $z T$. Such a nonplateau-like behavior is a characteristic of the free theory. However, within our present level of accuracy and an available range of $1<z T<2.5$, we could not identify a clear decrease of the effective screening masses with increasing $z T$ and have rather observed plateau-like behaviors. As an illustration, in Fig. 6 we show the effective screening masses in the pseudo-scalar channel as a function of $z T$ for a large range of temperatures. As can be seen from this figure, we have not found any clear evidence of a nonplateau-like behavior in the effective screening masses as seen in the free case (see Fig. 1). Moreover, the plateaulike structure in the effective screening masses are in good agreement with the values of the screening masses that have been obtained by fitting those correlators.

In the low temperature region of $0.75 T_{c} \lesssim T \lesssim T_{c}$ we have found that the PS screening mass remains almost constant (within $<10 \%$ ) staying close to the corresponding zero temperature mass and then starts rising rapidly. In this low $T$ regime also the $\mathrm{V}$ screening mass remains almost constant and then shows a rapid increase. On the other hand, the SC and the AV scalar screening masses show decreasing tendencies, attain minima around $T_{c}$ and then start rising again.

In the intermediate temperature regime $T_{c} \leq T \lesssim$ $1.5 T_{c}$ the spatial screening correlators show signals of chiral and effective $U_{A}(1)$ symmetry restorations. As discussed in Section 1. chiral symmetry restoration is reflected through the degeneracy of the $\mathrm{V}$ and AV correlation functions at all distances giving rise to degenerate $\mathrm{V}$ and AV screening masses. We found that, for $N_{\tau}=6, M_{V}$ becomes approximately equal to $M_{A V}$ at a temperature $T \simeq 200 \mathrm{MeV}$. This temperature is approximately equal to the chiral transition temperature $T_{c}$ for these lattices identified via the peak of the disconnected chiral susceptibility. In order to gain some more insight into this degeneracy in Fig. 7(a) we show the correlation functions of the $\mathrm{V}$ and $\mathrm{AV}$ channels at $T=203 \mathrm{MeV}$, i.e. $T \simeq 1.1 T_{c}$, for the $N_{\tau}=6$ lattice. Indeed we see that the signature of the chiral symmetry restoration is not only limited to the degeneracy of the screening masses, which are governed by the long distance behavior of the correlation functions, but also is reflected in the degeneracy between the correlation functions at very short distance scales $z T<1$. Similarly, as also mentioned in Section 1, the spatial correlation functions of the PS and the (iso-triplet) SC show effective restoration of $U_{A}(1)$ for the $2+1$ flavor theory. In the present work we have found that the PS and SC correlation functions become degenerate, and hence also the respective screening masses (see Fig. $3(\mathrm{c})]$, for $T \gtrsim 1.3 T_{c}$ only. To illustrate this fact in detail we show comparisons of the $\mathrm{PS}$ and $\mathrm{SC}$ screening correlators at $T \simeq 1.2 T_{c}$ in Fig. 7 (b) and at $T \simeq 1.3 T_{c}$ in Fig. 7(c). While at $T \simeq 1.2 T_{c}$ the PS and SC screening correlators start differing for distances $z T \gtrsim 0.5$ the correlators become degenerate at all distance scales, even for $z T<1$, at $T \simeq 1.3 T_{c}$. Moreover, as shown in Fig. 3(c), the magnitude of the mass splitting $\left(M_{S C}-M_{P S}\right)$ in the temperature range $T_{c} \lesssim T \lesssim 1.3 T_{c}$ remains almost unchanged in going from $\bar{u} d$ flavor content to $\bar{u} s$. This indicates that in this temperature regime the mass splitting $\left(M_{S C}-M_{P S}\right)$, which is sensitive to the presence of topologically non-trivial configurations [2], remains non-vanishing in the limit of mass-less light quarks. This conveys the point that the effective $U_{A}(1)$ restoration in QCD does not happen at the chiral transition temperature $T_{c}$, i.e. the temperature where the $\mathrm{V}$ and AV correlation functions become degenerate. This observation may have implications in relation to the possible existence of a first order chiral transition in the limit of two mass-less flavors [27].

As has been pointed out previously in Section 1, at higher temperatures $T>1.5 T_{c}$ the mesonic screening masses play an important role in testing the applicability of the dimensionally reduced perturbation theory. At these temperatures we have observed that the screening masses in the PS (SC) channel are always lower than the $\mathrm{V}(\mathrm{AV})$ screening masses. In other words, the largest spatial correlation length belongs to the PS (SC) channel. In order for the dimensional reduction to work this PS spatial correlation length must be smaller than the smallest spatial correlation length associated with a glueball like object. As can be seen from Fig. 8(a), $M_{P S} / T$ reaches values greater than 4 at $T \simeq 1.5 T_{c}$ and greater than 5 at $T \simeq 2 T_{c}$ for all three lattice spacings. Thus, already for $T \sim 1.5 T_{c}$ the value of the 'pion' screening mass is larger than the smallest glueball screening mass estimated in Ref. 4] and larger than the Debye mass estimated in Ref. [28. Hence, the lightest fermionic mode is comparatively heavy already at rather small temperatures such that dimensionally reduced QCD may work down to temperatures as low as about $1.5 T_{c}$, as was demonstrated for a quantity like the spatial string tension 5,6. This is in 


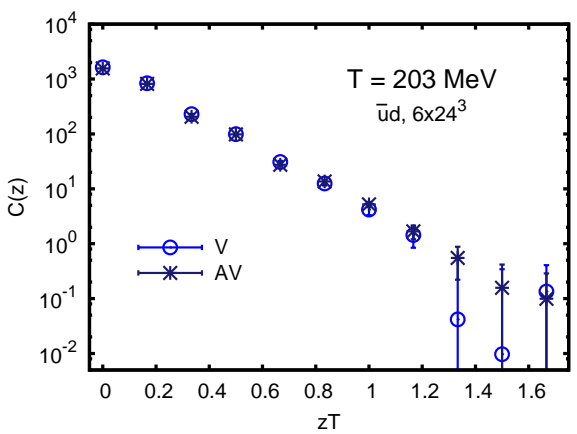

(a)

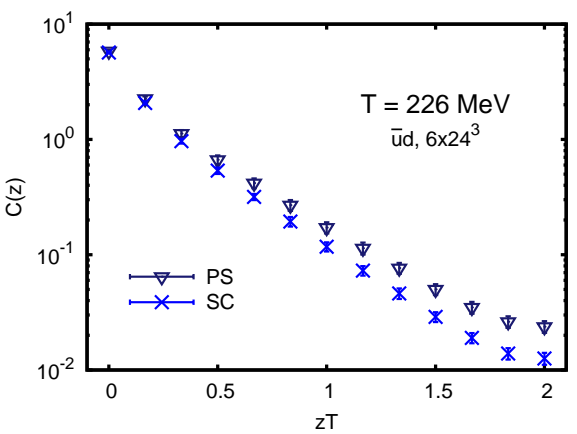

(b)

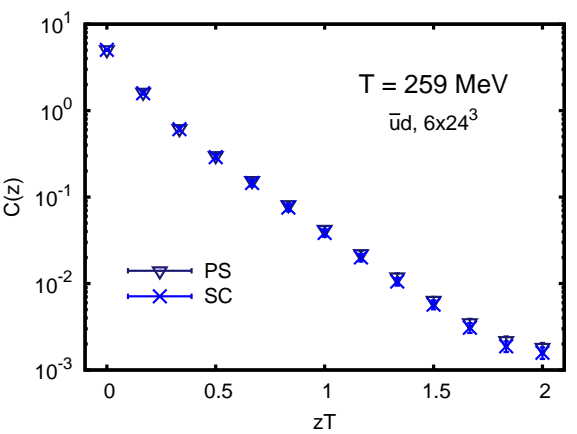

(c)

Fig. 7. Comparisons of spatial correlation functions for the $\bar{u} d$ flavor combination on $N_{\tau}=6$ lattices: (a) Vector and the axial-vector correlation functions at $T=203 \mathrm{MeV}$ using wall sources. The axial-vector correlation function has been multiplied by the factor $-(-1)^{z}$ to make it positive for each value of $z$. Pseudo-scalar and scalar correlation functions at $T=226 \mathrm{MeV}$ (b) and at $T=259 \mathrm{MeV}$ (c) using point sources. The scalar correlation function has been multiplied by $-(-1)^{z}$.

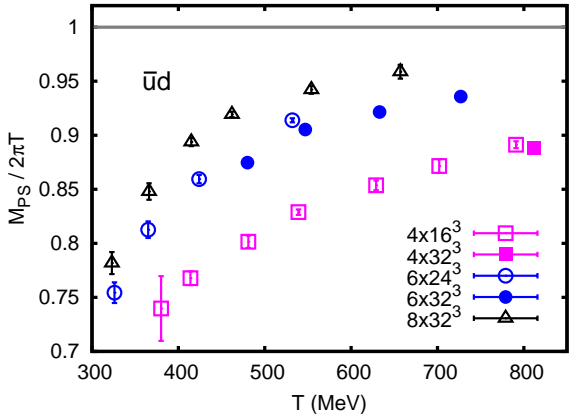

(a)

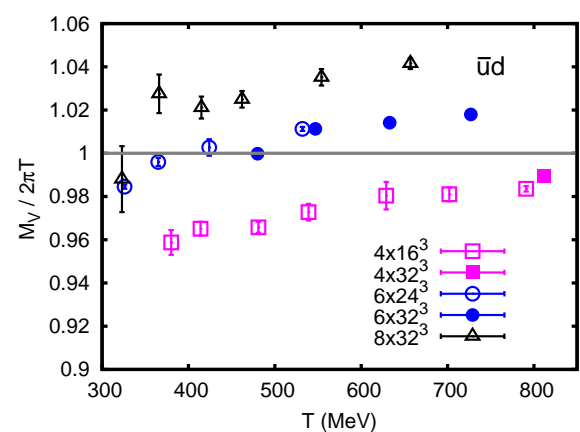

(b)

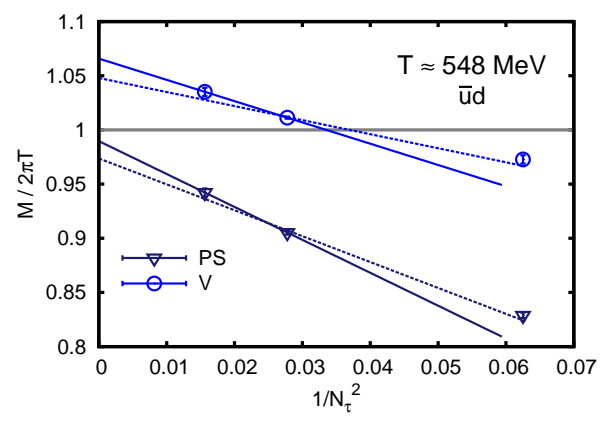

(c)

Fig. 8. Deviation of the pseudo-scalar (a) and vector (b) screening masses from the free (continuum) result of $2 \pi T$ for the $\bar{u} d$ sector. (c) Extrapolations, linear in $1 / N_{\tau}^{2}$, of the PS and V screening masses to zero lattice spacing at $T=548(1) \mathrm{MeV}$. The solid lines indicate extrapolations using only $N_{\tau}=6$ and 8 data. The dashed lines indicate the extrapolations when $N_{\tau}=4$ data are also included in the extrapolations.

contrast to the previous findings of Ref. 4 where it was concluded that the dimensional reduction framework may fail for $T \lesssim 3 T_{c}$.

In Figs. 8(a) and 8(b) we show close-up views of how the PS and the $\mathrm{V}$ screening masses deviate from their continuum free value of $2 \pi T$ in the high temperature region $T>1.5 T_{c}$. In this temperature regime for the pseudoscalar (and scalar) channel the ratio $M_{P S} / 2 \pi T$ not only rises rapidly towards 1 with increasing temperature, but also comes closer towards 1 with decreasing lattice spacing at a fixed temperature. For example, while on our coarsest lattice $M_{P S}$ deviates from $2 \pi T$ by about $10 \%$ at a temperature as high as $4 T_{c}$ this deviation becomes $\sim 5 \%$ for our finest lattice already at $T \simeq 3 T_{c}$. On the other hand, the ratio $M_{V} / 2 \pi T$ shows a rather slow increase with increasing temperature. While this ratio remains $\sim 2 \%$ below 1 at $T \simeq 4 T_{c}$ for our coarsest lattice spacing it becomes $\sim 4 \%$ larger than 1 at $T \simeq 3 T_{c}$ for our finest lattice spacing. In view of this, in Fig. 8(c), we attempt linear in $a^{2} \sim 1 / N_{\tau}^{2}$ extrapolations of the PS and $\mathrm{V}$ screening masses at a temperature $T \simeq 3 T_{c}$, i.e. the maximum temperature where we have data at all three lattice spacings. The dashed lines in Fig. 8(c) show extrapolations when all the three lattice spacings are used during the extrapolations. Such extrapolations give that the PS and the $\mathrm{V}$ screening masses, extrapolated to zero lattice spacing, are respectively $2-3 \%$ below and $5-6 \%$ above the free theory value of $2 \pi T$. As even at this high temperature the lattice spacing corresponding to $N_{\tau}=4$ is still quite large (about $0.09 \mathrm{fm}$ ) it is not clear whether including these data in our zero lattice spacing extrapolations yields reliable results. In order to have some quantitative idea on the systematics we have also performed extrapolations linear in $1 / N_{\tau}^{2}$ using only $N_{\tau}=6$ and 8 data. These are indicated by the solid lines in Fig. 8(c) Such extrapolations give that the continuum PS screening mass at this temperature may even be closer to $2 \pi T$, smaller only by $1 \%$, and the continuum $\mathrm{V}$ screening mass is about $7 \%$ larger than $2 \pi T$.

Perturbation theory predicts [8,9] that at high temperatures the screening masses of all the mesons are degenerate and larger than the free theory value of $2 \pi T$. Although we have found that the continuum extrapolated PS screening mass approaches the continuum free theory value of $2 \pi T$ very closely around $T \simeq 3 T_{c}$ and also, for 


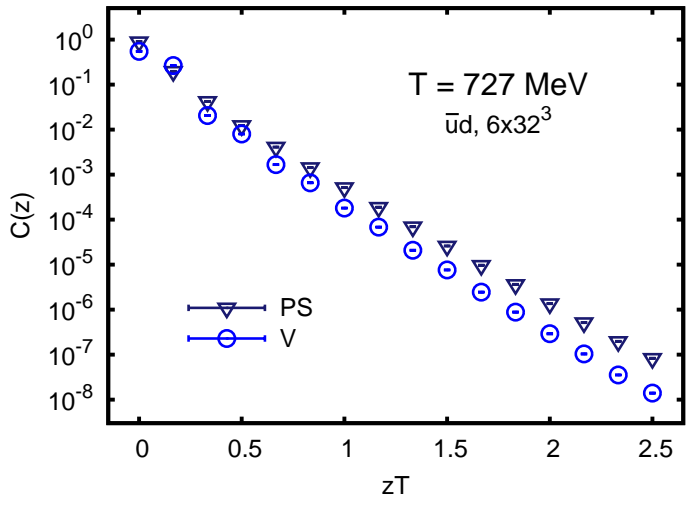

Fig. 9. A comparison of pseudo-scalar and vector correlation functions, obtained by using wall sources, on the $N_{\tau}=6$ lattice at $T=727 \mathrm{MeV}$.

the first time, found evidence that the $\mathrm{V}$ screening masses probably become larger than $2 \pi T$ at high temperature, we have not found any evidence that $M_{P S}$ and $M_{V}$ become degenerate up to temperatures at least as high as $T \simeq 4 T_{c}$. In order to have a closer look at this non-degeneracy, in Fig. 9 we compare directly the PS and V correlation functions as function of the distance $z T$ around $T \simeq 4 T_{c}$. It is clear from this comparison that the spatial correlators in the PS and $\mathrm{V}$ channels differ from each other at all distances including short ones of $z T<1$. In contrast, in the (mass-less) free theory the PS and V correlation functions themselves are degenerate at all distances even on a finite lattice. Although this observation is subject to cutoff and finite volume effects which could, in principle, be very different between the free and the interacting theory, we take it as a strong indication that the non-degeneracy of $M_{P S}$ and $M_{V}$ is probably not a lattice artifact and may arise due to the presence of spin dependent interactions, as suggested e.g. in 29, at the investigated temperatures.

As mentioned earlier, one of the sources of systematic errors in our study is the limited value of the maximum accessible distance $z T$. We have also found moderate lattice spacing dependence of screening masses at high temperature. Hence, it is important to corroborate our findings by performing analyses on lattices with larger aspect ratios and finer lattice spacings. We hope to address this issue in the future.

\section{Acknowledgments}

This work has been supported in part by contracts DEAC02-98CH10886 and DE-FG02-92ER40699 with the U.S. Department of Energy, the Bundesministerium für Bildung und Forschung under grant 06BI401, the Gesellschaft für Schwerionenforschung under grant BILAER, the Helmholtz Alliance HA216/EMMI grant and the Deutsche Forschungsgemeinschaft under grant GRK 881. The numerical computations have been carried out on the apeNEXT at Bielefeld University, the QCDOC computer of the RIKEN $\mathrm{BNL}$ research center, the DOE funded QCDOC at BNL and the BlueGene/L at the New York Center for Computational Sciences (NYCCS).

\section{Appendix}

Tables 3, 4 and 5] summarize the meson screening masses for the scalar, pseudo-scalar, averaged transverse axialvector and averaged transverse vector channels for the lightest $\bar{u} d$ flavor combination and for all the lattices that have been analyzed in this paper. The screening masses are given in lattice units. The data can be easily converted to either temperature or vacuum $\left(r_{0}\right)$ units by means of the included zero temperature results for $r_{0} / a$ from $[22$.

\section{References}

1. C. DeTar and J. B. Kogut, Phys. Rev. Lett. 59 (1987) 399, Phys. Rev. D36 (1987) 2828.

2. E. V. Shuryak, Comments Nucl. Phys. 21 (1994) 235; S. H. Lee and T. Hatsuda, Phys. Rev. D54 (1996) 1871; N. Evans, S. D. H. Hsu and M. Schwetz, Phys. Lett. B375 (1996) 262; M. C. Birse, T. D. Cohen and J. A. McGovern, Phys. Lett. B388 (1996) 137, Phys. Lett. B399 (1997) 263; J. B. Kogut, J. F. Lagaë and D. K. Sinclair, Phys. Rev. D58 (1998) 054504; G. V. Dunne and A. Kovner, Phys. Rev. D82 (2010) 065014.

3. T. Appelquist and R. D. Pisarski, Phys. Rev. D23 (1981) 2305.

4. R. Gavai and S. Gupta, Phys. Rev. Lett. 85 (2000) 2068.

5. M. Cheng et al., Phys. Rev. D78 (2008) 034506.

6. M. Laine and Y. Schröder, JHEP 03 (2005) 067; PoS LAT2005 (2006) 180.

7. W. Florkowski and B. Friman, Z. Phys. A347 (1994) 271.

8. M. Laine and M. Vepsäläinen, JHEP 02 (2004) 004.

9. W. M. Alberico, A. Beraudo, A. Czerska, P. Czerski and A. Molinari, Nucl. Phys. A792 (2007) 152.

10. F. Karsch and E. Laermann, in Quark gluon plasma III, R. Hwa (ed.), hep-lat/0305025.

11. R. Gavai and S. Gupta, Phys. Rev. D67 (2003) 034501.

12. S. Wissel et al., PoS LAT2005 (2006) 164.

13. E. Laermann, PoS LAT2008 (2008) 193.

14. R. Gavai, S. Gupta and R. Lacaze, Phys. Rev. D78 (2008) 014502 .

15. U. M. Heller, F. Karsch and B. Sturm, Phys. Rev. D60 (1999) 114502.

16. S. Mukherjee, PoS LAT2007 (2007) 210, Nucl. Phys. A820 (2009) 283c, PoS Confinement8 (2009) 116.

17. H. Kluberg-Stern et al., Nucl. Phys. B220 (1983) 447.

18. M. F. L. Golterman, Nucl. Phys. B273 (1986) 663.

19. R. Altmeyer et al., Nucl. Phys. B389 (1993) 445.

20. S. Gupta, Phys. Rev. D60 (1999) 094505.

21. I. Horváth, A. D. Kennedy and S. Sint, Nucl. Phys. Proc. Suppl. 73 (1999) 834; M. A. Clark, A. D. Kennedy and Z. Sroczynski, Nucl. Phys. Proc. Suppl. 140 (2005) 835; M. A. Clark and A. D. Kennedy, Phys. Rev. Lett. 98 (2007) 051601.

22. M. Cheng et al., Phys. Rev. D77 (2008) 014511.

23. A. Bazavov et al., Phys. Rev. D80 (2009) 014504.

24. R. Sommer, Nucl. Phys. B411 (1994) 839.

25. A. Gray et al., Phys. Rev. D72 (2005) 094507. 


\begin{tabular}{|c|c|c|c|c|c|}
\hline$\beta$ & $r_{0} / a$ & $a M_{S C}$ & $a M_{P S}$ & $a M_{A V}$ & $a M_{V}$ \\
\hline \multicolumn{6}{|c|}{$16^{3} \times 4$} \\
\hline \multicolumn{6}{|c|}{ point sources } \\
\hline 3.290 & 1.823 & $0.860(80)$ & $0.293(08)$ & & \\
\hline 3.320 & 1.908 & $0.702(20)$ & $0.349(08)$ & & \\
\hline 3.351 & 2.069 & $0.695(18)$ & $0.517(08)$ & $1.33(12)$ & $1.37(21)$ \\
\hline 3.382 & 2.230 & $0.802(11)$ & $0.778(15)$ & $1.28(10)$ & $1.33(12)$ \\
\hline 3.410 & 2.503 & $0.920(10)$ & $0.902(18)$ & $1.46(06)$ & $1.46(06)$ \\
\hline 3.460 & 2.890 & $1.046(48)$ & $1.043(46)$ & $1.54(09)$ & $1.52(08)$ \\
\hline 3.540 & 3.687 & $1.162(36)$ & $1.162(47)$ & $1.48(05)$ & $1.49(06)$ \\
\hline 3.570 & 4.009 & $1.206(10)$ & $1.206(10)$ & $1.53(01)$ & $1.53(01)$ \\
\hline 3.630 & 4.651 & $1.259(09)$ & $1.259(09)$ & $1.54(01)$ & $1.54(01)$ \\
\hline 3.690 & 5.201 & $1.306(08)$ & $1.302(04)$ & $1.55(01)$ & $1.55(01)$ \\
\hline 3.760 & 6.050 & $1.341(07)$ & $1.341(07)$ & $1.56(02)$ & $1.57(02)$ \\
\hline 3.820 & 6.752 & $1.369(10)$ & $1.369(10)$ & $1.57(02)$ & $1.57(02)$ \\
\hline 3.920 & 7.590 & $1.400(05)$ & $1.400(05)$ & $1.56(01)$ & $1.56(01)$ \\
\hline \multicolumn{6}{|c|}{ wall sources } \\
\hline 3.382 & 2.230 & $0.801(13)$ & $0.762(19)$ & $1.380(60)$ & $1.380(50)$ \\
\hline 3.410 & 2.503 & $0.905(11)$ & $0.890(13)$ & $1.460(60)$ & $1.450(70)$ \\
\hline 3.460 & 2.890 & $1.054(22)$ & $1.051(22)$ & $1.515(35)$ & $1.520(38)$ \\
\hline 3.540 & 3.687 & $1.166(10)$ & $1.166(10)$ & $1.506(09)$ & $1.506(09)$ \\
\hline 3.570 & 4.009 & $1.204(06)$ & $1.204(06)$ & $1.516(05)$ & $1.516(05)$ \\
\hline 3.630 & 4.651 & $1.255(05)$ & $1.255(05)$ & $1.517(04)$ & $1.517(04)$ \\
\hline 3.690 & 5.201 & $1.291(05)$ & $1.291(05)$ & $1.528(06)$ & $1.528(06)$ \\
\hline 3.760 & 6.050 & $1.330(04)$ & $1.330(04)$ & $1.540(10)$ & $1.540(10)$ \\
\hline 3.820 & 6.752 & $1.355(04)$ & $1.355(04)$ & $1.541(05)$ & $1.541(05)$ \\
\hline 3.920 & 7.590 & $1.386(02)$ & $1.386(02)$ & $1.545(02)$ & $1.545(02)$ \\
\hline \multicolumn{6}{|c|}{$32^{3} \times 4$} \\
\hline \multicolumn{6}{|c|}{ point sources } \\
\hline 3.920 & 7.814 & & $1.395(2)$ & & $1.560(1)$ \\
\hline \multicolumn{6}{|c|}{ wall sources } \\
\hline 3.920 & 7.814 & & $1.392(1)$ & & $1.554(2)$ \\
\hline
\end{tabular}

Table 3. Screening masses from $N_{\tau}=4$ lattices.

26. C. Bernard et al., Phys. Rev. D76 (2007) 094504.

27. R. D. Pisarski and F. Wilczek, Phys. Rev. D29 (1984) 338; F. Basile, A. Pelissetto and E. Vicari, PoS LAT2005 (2006) 199; S. Chandrasekharan and A. C. Mehta, Phys. Rev. Lett. 99 (2007) 142004; M. D'Elia, A. D. Giacomo and C. Pica, Phys. Rev. D72 (2005) 114510.

28. K. Petrov, PoS LAT2007 (2007) 217; O. Kaczmarek, PoS CPOD07 (2007) 048.

29. V. Koch et al., Phys. Rev. D46 (1992) 3169. 


\begin{tabular}{|c|c|c|c|c|c|}
\hline$\beta$ & $r_{0} / a$ & $a M_{S C}$ & $a M_{P S}$ & $a M_{A V}$ & $a M_{V}$ \\
\hline \multicolumn{6}{|c|}{$24^{3} \times 6$} \\
\hline \multicolumn{6}{|c|}{ point sources } \\
\hline 3.351 & 2.069 & $0.900(30)$ & $0.257(01)$ & & \\
\hline 3.410 & 2.503 & $0.700(20)$ & $0.226(02)$ & & \\
\hline 3.430 & 2.647 & $0.615(09)$ & $0.216(03)$ & & \\
\hline 3.445 & 2.813 & $0.510(20)$ & $0.253(05)$ & & \\
\hline 3.455 & 2.856 & $0.420(10)$ & $0.292(07)$ & & \\
\hline 3.460 & 2.890 & $0.435(08)$ & $0.334(08)$ & & \\
\hline 3.490 & 3.223 & $0.490(10)$ & $0.440(10)$ & & \\
\hline 3.510 & 3.426 & $0.580(10)$ & $0.560(10)$ & & \\
\hline 3.540 & 3.687 & $0.648(09)$ & $0.640(08)$ & $1.11(6)$ & $1.08(6)$ \\
\hline 3.570 & 4.009 & $0.728(08)$ & $0.726(08)$ & $1.04(3)$ & $1.04(3)$ \\
\hline 3.630 & 4.651 & $0.790(10)$ & $0.790(10)$ & $1.04(1)$ & $1.04(2)$ \\
\hline 3.690 & 5.201 & $0.851(08)$ & $0.851(08)$ & $1.04(1)$ & $1.04(1)$ \\
\hline 3.760 & 6.050 & $0.900(04)$ & $0.900(04)$ & $1.06(1)$ & $1.06(1)$ \\
\hline 3.920 & 7.590 & $0.957(02)$ & $0.957(02)$ & $1.07(1)$ & $1.07(1)$ \\
\hline \multicolumn{6}{|c|}{ wall sources } \\
\hline 3.351 & 2.069 & $1.070(50)$ & $0.270(3)$ & $1.650(20)$ & $1.270(30)$ \\
\hline 3.410 & 2.503 & $0.780(20)$ & $0.240(2)$ & $1.408(09)$ & $1.125(05)$ \\
\hline 3.430 & 2.647 & $0.685(02)$ & $0.227(1)$ & $1.140(50)$ & $0.985(06)$ \\
\hline 3.445 & 2.813 & $0.512(02)$ & $0.246(1)$ & $1.090(04)$ & $0.994(04)$ \\
\hline 3.455 & 2.856 & $0.456(02)$ & $0.262(2)$ & $1.058(05)$ & $0.990(10)$ \\
\hline 3.460 & 2.890 & $0.436(01)$ & $0.316(1)$ & $1.010(08)$ & $1.030(10)$ \\
\hline 3.490 & 3.223 & $0.487(01)$ & $0.438(1)$ & $0.990(06)$ & $0.990(07)$ \\
\hline 3.510 & 3.426 & $0.562(01)$ & $0.538(2)$ & $1.009(04)$ & $1.002(04)$ \\
\hline 3.570 & 4.009 & $0.705(01)$ & $0.702(1)$ & $1.016(01)$ & $1.011(01)$ \\
\hline 3.630 & 4.651 & $0.788(01)$ & $0.788(1)$ & $1.031(01)$ & $1.031(01)$ \\
\hline 3.690 & 5.201 & $0.839(01)$ & $0.839(1)$ & $1.043(02)$ & $1.043(02)$ \\
\hline 3.760 & 6.050 & & & $1.049(05)$ & $1.050(04)$ \\
\hline 3.920 & 7.590 & $0.938(01)$ & $0.941(1)$ & $1.059(01)$ & $1.059(01)$ \\
\hline \multicolumn{6}{|c|}{$32^{3} \times 6$} \\
\hline \multicolumn{6}{|c|}{ point sources } \\
\hline 3.820 & 6.864 & & $0.916(3)$ & & $1.059(4)$ \\
\hline 3.920 & 7.814 & & $0.948(2)$ & & $1.061(4)$ \\
\hline 4.000 & 9.048 & & $0.965(3)$ & & $1.063(4)$ \\
\hline 4.080 & 10.390 & & $0.980(3)$ & & $1.064(2)$ \\
\hline \multicolumn{6}{|c|}{ wall sources } \\
\hline 3.820 & 6.864 & & $0.911(2)$ & & $1.047(2)$ \\
\hline 3.920 & 7.814 & & $0.946(1)$ & & $1.059(1)$ \\
\hline 4.000 & 9.048 & & $0.961(1)$ & & $1.062(2)$ \\
\hline 4.080 & 10.390 & & $0.978(2)$ & & $1.066(2)$ \\
\hline
\end{tabular}

Table 4. Screening masses from $N_{\tau}=6$ lattices. 


\begin{tabular}{|rr|cccc|}
\hline$\beta$ & $r_{0} / a$ & $a M_{S C}$ & $a M_{P S}$ & $a M_{A V}$ & $a M_{V}$ \\
\hline \hline \multicolumn{7}{|c|}{$32^{3} \times 8$} \\
\hline \multicolumn{7}{|c|}{ point sources } \\
\hline 3.430 & 2.647 & $0.201(01)$ & & \\
3.500 & 3.328 & $0.463(174)$ & $0.175(01)$ & & \\
3.570 & 4.009 & $0.366(010)$ & $0.311(14)$ & & \\
3.585 & 4.160 & $0.382(010)$ & $0.351(10)$ & & \\
3.760 & 6.050 & $0.615(008)$ & $0.614(08)$ & $0.777(12)$ & $0.776(12)$ \\
3.820 & 6.864 & $0.666(006)$ & $0.666(06)$ & $0.807(07)$ & $0.807(07)$ \\
3.920 & 7.814 & $0.702(003)$ & $0.702(03)$ & $0.802(04)$ & $0.802(04)$ \\
4.000 & 9.048 & $0.721(001)$ & $0.722(02)$ & $0.803(02)$ & $0.805(03)$ \\
4.080 & 10.390 & $0.740(003)$ & $0.740(03)$ & $0.813(03)$ & $0.813(03)$ \\
4.200 & 12.420 & $0.753(006)$ & $0.753(05)$ & $0.831(12)$ & $0.816(09)$ \\
\hline \multicolumn{7}{|c|}{ wall sources } \\
\hline 4.000 & \multicolumn{7}{|c|}{$0.716(001)$} & $0.716(03)$ & $0.799(04)$ & $0.800(03)$ \\
4.200 & $0.755(002)$ & $0.755(01)$ & $0.832(12)$ & $0.818(02)$ \\
\hline
\end{tabular}

Table 5. Screening masses from $N_{\tau}=8$ lattices. 